\title{
Operative site irrigation with povidone-iodine solution in spinal surgery for surgical site infection prevention: Can it be used safety?
}

\author{
Reza Akhavan Sigari ${ }^{1}$, Hesam Abdolhoseinpour ${ }^{2}$ \\ 1-Department of Neurosurgery, University Medical Center Tuebingen, Eberhard-Karls University, Tuebingen, Germany. \\ 2-Department of Neurosurgery, Bou Ali Hospital, Faculty of Medicine, Tehran Medical Sciences Branch, Islamic Azad University, \\ Tehran, Iran.
}

Correspondence: Hesam Abdolhoseinpour; E-mail: abdolhosseinpoor@yahoo.com; Tel: 00989126306851

\section{Abstract}

Background: Povidone-iodine (PVI) is an effective disinfection solution for surgical wounds. However, there is some reports of its adverse effects on wound healing and bone reunion. Here, we evaluate the efficacy and safety of PVI irrigation in the prevention of surgical site infection in spinal surgery.

Methodology: From February 2011 to June 2019, 936 candidates of spinal fusion surgery were randomized to receive PVI 3\% ( $\mathrm{n}=$ 468 ) or normal saline (NS) irrigation ( $n=468$ ) using convenient sampling. Before bone grafting, the surgical wounds were irrigated with NS $0.9 \%$ or PVI $3 \%$ followed by NS for a maximum of two min. The rate of postoperative surgical site infection, wound dehiscence, vertebral fusion, pain intensity, and function score were evaluated. The pain was scored using the visual analogue scale (VAS).

Results: The wound infection rate was significantly less in the PVI group compared with the NS group [5 (1.1\%) vs. 21(4.48\%), $p=$ 0.032]. There was no significant difference in the rate of vertebral union or wound dehiscence between the two groups. The pain VAS and function scores were both improved after surgery while this improvement was comparable between the study groups.

Conclusion: Lavage of surgical wounds with povidone-iodine $3 \%$ prevents surgical site infection in spinal surgeries without influencing wound healing, bone union or the clinical outcome.

Key words: Intraoperative irrigation; Povidone-iodine solution; Surgery, Spinal.

Citation: Sigari RA, Abdolhoseinpour H. Operative site irrigation with povidone-iodine solution in spinal surgery for surgical site infection prevention: Can it be used safety? Anaesth. pain intensive care 2020;24(3):314-319.

Received: 13 March 2020, Reviewed: 14 May 2020, Revised: 20 May 2020, Accepted: 27 May 2020

\section{Introduction}

Prevention of surgical site infection (SSI) following spinal operations requires a multifaceted approach, including preoperative parenteral antibiotic use and intraoperative wound irrigation with or without disinfectants, which help to remove body fluids, cellular debris, and microbes. ${ }^{1-3}$ Choice of an appropriate solution has always been a debatable issue. Normal saline, antibiotic or povidone-iodine (PVI) solutions are the most commonly used solutions for wound irrigation. ${ }^{4}$
The recent practice guidelines by the Centers for Disease Control \& Prevention (CDC) of the Department of Health and Human Services, USA, the National Institute for Health and Care Excellence (NICE), and the World Health Organization (WHO) have recommended the use of aqueous PVI for skin preparation before surgical incision as well as the incisional wound irrigation before closure in orthopedic spine surgery to prevent SSI. ${ }^{1-3}$ Intraoperative use of dilute PVI for clean and clean-contaminated wounds irrigation has 
been suggested by the second International Consensus Meeting on Musculoskeletal Infection, too. ${ }^{5}$ However, there have been some reports of PVI associated potential adverse effects that seem to depend on its concentration and duration of exposure. Systemic iodine toxicity has been found following PVI irrigation in historical case studies. ${ }^{6,7}$ Furthermore, in vitro studies show the negative effects of PVI on tissue regeneration, embryonic chick osteoblasts, and cultured chondrocytes. ${ }^{8-}$ ${ }^{10}$ Experimental exposure of rat spinal cord to $0.1 \%$ PVI after laminectomy-durotomy caused neurotoxicity. ${ }^{11}$ Therefore, an optimal balance needs to be established between PVI bactericidal effects and cytotoxicity. There is limited data on the safety dose spectrum of PVI for spinal surgeries. ${ }^{12-14}$ On the other hand, normal saline has the lowest toxicity, but it only dilutes and does not cleanse the contaminants. ${ }^{13}$

We conducted the current randomized clinical trial aimed to evaluate the effect of wound irrigation with PVI and normal saline on infection rate, wound healing, fusion status and clinical outcomes of spinal surgery.

\section{Methodology}

It was a randomized, blinded trial in which the eligible patients were randomized to two groups; either to receive wound irrigation by $0.9 \%$ normal saline (NS) or PVI 3.5\% solution.

The study was approved by institutional ethical committee. The inclusion criteria were candidates undergoing instrumented thoracic/lumbar spinal fusion surgery due to scoliosis or other degenerative diseases reporting to Bouali Hospital, Tehran, Iran, between February 2011 and June 2019. A total of 936 consecutive patients were included. The exclusion criteria were a history of PVI allergy, clinical signs of infection before surgery, dura laceration, implant removal surgery, or prior spinal surgery. Any risk factors for infection such as diabetes mellitus, rheumatoid arthritis, cancer, and daily use of steroids or immunosuppressants were recorded.

Informed consent was obtained from each patient. The study was performed according to the Declaration of Helsinki and the Medical Research Involving Human Subjects. ${ }^{15}$

All the participants received the routine peri-operative care for infection control. Inj. cefuroxime $(3000 \mathrm{mg}$
IV) was routinely administered an hour before the operation as a preoperative prophylactic, and additional doses of inj. cefuroxime were repeated every $12 \mathrm{~h}$ for $48 \mathrm{~h}$ postoperatively. The surgical site was disinfected and prepped with sterile drapes then covered with disposable drapes. A standard midline incision and an open approach were used. All the surgeries, except the ventral cervical instrumentation, were performed by a posterior central surgical access through a $37-61 \mathrm{~cm}$ incision. All the surgeries were performed under the same standard operative environment and conditions, with the same surgical technique by the same surgical team.

Before bone grafting, the surgical wounds in the NS group were filled and soaked with $0.9 \% \mathrm{NS}$, suction was performed; the irrigation being repeated three times. In the PVI group, surgical wounds were irrigated with PVI 3\% to fill and soak the wound for two minutes followed by NS irrigation. Bone grafting was then performed with no more irrigation. The wound was closed in layers and suction drain was applied to be removed 48-72 $\mathrm{h}$ later, if the output was less than $50 \mathrm{ml} / \mathrm{d}$.

The SSI was diagnosed in accordance with the CDC criteria. ${ }^{1}$ Infection was suspected with fever and unusual surgical site pain. Consequently, WBC count, ESR, C-reactive protein, and microbiological cultures from the surgical wounds were requested. Confirmed superficial SSI was treated with antibiotics. Patients with deep SSI were re-operated for extensive debridement and irrigation, followed by antimicrobial treatments. All patients were followed up for at least 12 months regarding the rate of SSI infection, wound dehiscence, spinal bone fusion, and pain and function scores. The pain was evaluated by the visual analogue scale (VAS), which is a continuous scale comprised of a $10 \mathrm{~cm}$ horizontal line. ${ }^{16}$ The patients were asked to mark the VAS line at the point that represented their pain intensity in the last 2 weeks. The score was determined by measuring the distance (in $\mathrm{mm}$ ) between zero and the patient's mark. The interbody vertebral fusion was assessed by anteroposterior, lateral, and flexion/extension radiographs of the spine.

Investigators, who were blinded to the randomization method and assigned treatments, collected and analyzed the data. The laboratory staff was also kept unaware of the treatment assignment. Statistical analysis: Statistical analysis was done using SPSS 24 (SPSS 
Inc., IL, USA). P < 0.05 was considered statistically significant.

\section{Results}

All the 936 enrolled patients randomized to receive either PVI or NS wound irrigation completed the study. In both groups, about 55\% (256 in the PVI group and 261 in the NS group) patients were male. The mean age was 68.1 (range 31-82) y and 67.9 (range 27-90) y in the PVI and NS groups respectively. The PVI group included 316 cases / 982 fusion levels; and the NS group included 301 cases / 831 fusion levels. The demographic and operative data are summarized in Table 1.
There were $3(0.64 \%)$ superficial and $2(0.42 \%)$ deep wound infections in the PVI group $(\mathrm{p}=0.032)$. While $12(2.6 \%)$ cases of superficial and $9(1.9 \%)$ The wound infection rate was significantly less in the PVI group compared to NS group, e.g., $1.1 \%$ vs. $4.48 \%$ cases of deep infection were found in the NS group. Interestingly, the fusion rate between the groups was $94.4 \%$ vs. $91.6 \%$ in the PVI and NS groups respectively ( $\mathrm{p}=$ 0.051 ) the difference being not significant. The pain VAS score was significantly reduced in all the participants while the difference was comparable between the study groups. Wound dehiscence was noted in 3 $(0.64 \%)$ of patients in the PVI group and in $12(2.56 \%)$ of patients in the NS group ( $>>0.05)$. There was also no significant difference in function score between the groups (Table 2).

Table 1: Patients' characteristics and operational data

\begin{tabular}{|c|c|c|c|}
\hline Variables & PVI $(n=468)$ & NS $(n=468)$ & p value \\
\hline $\begin{array}{l}\text { Age }(y)^{*} \\
\text { (range) }\end{array}$ & $\begin{array}{c}68.1 \pm 9.3 \\
(31-82)\end{array}$ & $\begin{array}{c}67.9 \pm 8.8 \\
(27-90)\end{array}$ & 0.004 \\
\hline Sex (male); $\mathrm{n}(\%)$ & $256(54.7)$ & $261(55.7)$ & 0.56 \\
\hline Comorbidity factors**; n(\%) & $302(64.5)$ & $288(61.5)$ & 0.64 \\
\hline \multicolumn{4}{|l|}{ Instrumentation (n) } \\
\hline - Cervical & 34 & 41 & \\
\hline - Thoracic & 15 & 8 & \\
\hline - $\quad$ Total (Patients / levels fused) & $316 / 982$ & $301 / 831$ & 0.003 \\
\hline \multicolumn{4}{|l|}{ Without Instrumentation (n) } \\
\hline $\begin{array}{l}\text { - Spinal laminectomy/spinal } \\
\text { decompression }\end{array}$ & 68 & 45 & \\
\hline $\begin{array}{l}\text { - Lumbar and cervical disc her- } \\
\text { niation }\end{array}$ & 77 & 129 & 0.053 \\
\hline Operation time $(\mathrm{min})^{*}$ & $191.5 \pm 35.7$ & $188.8 \pm 38.4$ & 0.431 \\
\hline Intraoperative bleeding $(\mathrm{ml})^{*}$ & $412 \pm 189$ & $397 \pm 197$ & 0.168 \\
\hline
\end{tabular}

*Mean $\pm S D ;{ }^{* *}$ Diabetes mellitus, rheumatoid arthritis, cancer, immunocompromised state 
Table 2: Post-operative outcome measures

\begin{tabular}{|c|c|c|c|}
\hline Outcome & PVI $(n=468)$ & NS $(n=468)$ & $p$ value \\
\hline Infection rate [n (\%)] & $5(1.1)$ & $21(4.48)$ & \multirow{3}{*}{0.032} \\
\hline - $\quad$ Superficial infection & $3(0.64)$ & $12(2.6)$ & \\
\hline - Deep infection & $2(0.42)$ & $9(1.9)$ & \\
\hline Fusion rate [n (\%)] & $442(94.4)$ & $429(91.6)$ & 0.051 \\
\hline Wound dehiscence [n (\%)] & $3(0.64)$ & $12(2.56)$ & 0.27 \\
\hline VAS pain (mean \pm SD) & $1 \pm 0.6$ & $2 \pm 0.4)$ & 0.85 \\
\hline Function score (mean \pm SD) & $7.65 \pm 3.9$ & $7.1 \pm 4.1$ & 0.12 \\
\hline
\end{tabular}

\section{Discussion}

Our findings show that the infection rate was significantly reduced with PVI $3 \%$ wound irrigation for $2 \mathrm{~min}$ prior to bone grafting compared with NS only irrigation. While vertebral fusion rate, wound dehiscence, pain severity and function scores were comparable between the study groups.

The 3 min soak with PVI $0.35 \%$ following by an additional $2 \mathrm{~L}$ of NS is recommended to be used before bone grafting and spinal instrumentation in orthopedic spine surgeries. ${ }^{1-3}$ PVI is a chemical complex of polyvinylpyrrolidone and elemental iodine that is gradually released and is toxic to microorganisms. It has a broad-spectrum antiseptic effect against pathogens such as Staphylococcus epidermidis, Pseudomonas aeruginosa, Haemophilus influenzae, Burkholderia cepacia, Enterococcus faecium. ${ }^{1}$ Escherichia coli, methicillin-resistant Staphylococcus aureus (MRSA) and other antibiotic-resistant strains. ${ }^{5,17,18}$

Schmidt et al. recently suggested the use of PVI 10\% for $1 \mathrm{~min}$ or $3.5 \%$ for $10 \mathrm{~min}$ for $\mathrm{S}$ epidermidis eradication and biofilm penetration. ${ }^{19}$ Experimental studies have shown the neurotoxic effect of PVI $0.1 \%$ in rats due to hypoxic myelin/axonal degeneration. ${ }^{11}$ Accordingly, PVI has been avoided in wound irrigation over any neural structures. It seems that the cytotoxic effect of PVI depends on its concentration and duration of exposure. Recent clinical studies have shown that wound irrigation with PVI significantly reduces the infection rate in spinal surgeries without causing any serious side effects. ${ }^{12,13,20,21}$
While there is no consensus on the safe dose and irrigation time with PVI. J Strohecker et al. found that intra-lumbar disc surgery lavage of diluted PVI decreases the infection rate by $1.6 \%$; however, the concentration of PVI was not provided..$^{20}$ Another study showed a significantly lower infection rate with PVI $0.35 \%$ irrigation (0\%) compared with NS (4.8\%) in spine surgeries, while wound healing and bone union were negatively affected. The pain and function scores were also improved. ${ }^{12}$ Later, WF Sindelar et al. revealed that a $6.15 \%$ PVI- $\mathrm{H}_{2} \mathrm{O}_{2}$ mixture versus no lavage in spine surgeries significantly reduced the infection rate from $1.5 \%$ to $0 \% .{ }^{21}$ A recent study used a similar concentration of PVI (3.35\%) found that in comparison to NS irrigation it is effective in reducing SSI from $4.4 \%$ to $0 \%$ in spinal surgeries without impairing the vertebral fusion rate, wound healing, or function score. Moreover, they did not find any linear correlation between PVI soaking time and the fusion rate. $^{13}$

We found almost similar results with higher doses of PVI; however, the infection rate did not decrease to zero in the current experiment. In addition to wound irrigation, several patients, wounds, and environmental strategies are needed to prevent intra-operative wound contamination, ${ }^{5}$ which explains the dissimilarity of infection rates. Recently, $90 \mathrm{sec}$ of $1 \%$ PVP-I pooling every $1.5 \mathrm{~h}$ followed by saline irrigation has been suggested to be safe for the prevention of postoperative deep SSI after spinal surgery. ${ }^{14}$

\section{Conclusion}

The results of our study show that intra-operative irrigation with a 3\% PVI solution significantly reduces the 
post-surgery infection rate with no effect on the fusion rate, wound healing, pain intensity, or function compared to the saline only irrigation.

\section{Conflict of interest}

None declared by the authors

\section{Authors' contribution}

All authors took equal part in the conduct of this study and preparation of the manuscript.

\section{References}

1. Berríos-Torres SI, Umscheid CA, Bratzler DW, Leas B, Stone EC, Kelz RR, et al. Centers for disease control and prevention guideline for the prevention of surgical site infection, 2017. JAMA Surg. 2017;152(8):784-791. [PubMed] DOI: 10.1001/jamasurg.2017.0904

2. World Health Organization. Global guidelines for the prevention of surgical site infection. Geneva; 2017.

3. Excellence NIfHaC. Surgical site infection: prevention and treatment of surgical site infection. Clinical guideline 74. 2017.

4. Alamanda V, Springer B. The prevention of infection: 12 modifiable risk factors. Bone Joint J. 2019;101(1_Supppl_A):3-9. [PubMed] DOI: 10.1302/0301-620X.101B1.BJJ-2018-0233.R1

5. Goswami K, Austin MS. Intraoperative povidoneiodine irrigation for infection prevention. Arthroplasty today. 2019;5(3):306-308. [PubMed] DOI: $10.1016 /$ i.artd.2019.04.004

6. Lavelle KJ, Doedens DJ, Kleit SA, Forney RB. lodine absorption in burn patients treated topically with povidone-iodine. Clin Pharmacol Ther. 1975;17(3):355-362. [PubMed] DOI: 10.1002/cpt1975173355

7. Pietsch J, Meakins J. Complications of povidoneiodine absorption in topically treated burn patients. Lancet. 1976;307(7954):280-282. [PubMed] DOl: 10.1016/s0140-6736(76)91406-9

8. Kaysinger KK, Nicholson NC, Ramp WK, Kellam JF. Toxic effects of wound irrigation solutions on cultured tibiae and osteoblasts. J Orthop Trauma. 1995;9(4):303-311. [PubMed] DOI: 10.1097/00005131-199509040-00006

9. Cooper ML, Laxer JA, Hansbrough JF. The cytotoxic effects of commonly used topical antimicrobial agents on human fibroblasts and keratinocytes. J Trauma. 1991;31(6):775-782; discussion 82-84. [PubMed] DOI: 10.1097/00005373-199106000-00007

10. von Keudell A, Canseco JA, Gomoll AH. Deleterious effects of diluted povidone-iodine on articular cartilage. J Arthroplasty. 2013;28(6):918291. [PubMed] DOI: 10.1016/].arth.2013.02.018

11. Akcay E, Ersahin $Y$, Ozer F, Duransoy YK, Camlar $M$, Atci I, et al. Neurotoxic effect of povidoneiodine on the rat spine using a laminectomydurotomy model. Childs Nerv Syst. 2012;28(12):2071-2075. [PubMed] DOl: 10.1007/s00381-012-1885-7

12. Chang FY, Chang MC, Wang ST, Yu WK, Liu CL, Chen $\mathrm{TH}$. Can povidone-iodine solution be used safely in a spinal surgery? Eur Spine J. 2006 Jun;15(6):1005-1014. [PubMed] DOI: 10.1007/s00586-005-0975-6

13. Allam AF, Abotakia TA, Farghally MY. Povidonelodine $3.35 \%$ solution versus normal saline for irrigation in lumbar fusion surgery: a retrospective study. Egyptian Spine J. 2019;30(1):48-56. DOI: 10.21608/esj.2019.5477.1069

14. Onishi Y, Masuda K, Tozawa K, Karita T. Outcomes of an intraoperative povidone-iodine irrigation protocol in spinal surgery for surgical site infection prevention. Clin Spine Surg. 2019 Dec;32(10):E449-E452. [PubMed] DOI: 10.1097/BSD.0000000000000908

15. World Medical Association. World Medical Association Declaration of Helsinki: ethical principles for medical research involving human subjects. JAMA. 2013 Nov 27;310(20):2191-2194. [PubMed] DOI: 10.1001/jama.2013.281053

16. Jensen MP, Karoly P, Braver S. The measurement of clinical pain intensity: a comparison of six methods. Pain. 1986;27(1):117-126. [PubMed] DOI: 10.1016/0304-3959(86)90228-9

17. Oduwole KO, Glynn AA, Onayemi FO, Molony D, Gara JP, McCormack D. (eds.) Anti-biofilm activity of subinhibitory povidone-iodine concentrations against staphylococcus epidermidis and staphylococcus aureus. Orthopaedic Proceedings; 2011.

18. Reimer K, Wichelhaus $T$, Schäfer V, Rudolph $P$, Kramer A, Wutzler $P$, et al. Antimicrobial effectiveness of povidone-iodine and consequences for new application areas. Dermatology. 2002;204(Suppl 1):114-120. [PubMed] DOI: 10.1159/000057738

19. Schmidt K, Estes C, McLaren A, Spangehl MJ. Chlorhexidine antiseptic irrigation eradicates saphylococcus epidermidis from biofilm: an in vitro study. Clin Orthop Relat Res. 2018 Mar;476(3):648-653. [PubMed] DOI: $\underline{10.1007 / s 11999.0000000000000052}$ 
20. Strohecker J, Piotrowski W, Lametschwandtner A. The intra-operative application of povidone-iodine in neurosurgery. J Hosp Infect. 1985;6:123-125. [PubMed] DOI: 10.1016/s0195-6701(85)80057-8
21. Sindelar W, Mason G. (eds.) Efficacy of povidoneiodine irrigation in prevention of surgical wound infections. Surgical forum; 1977.

\section{The Anesthetist}

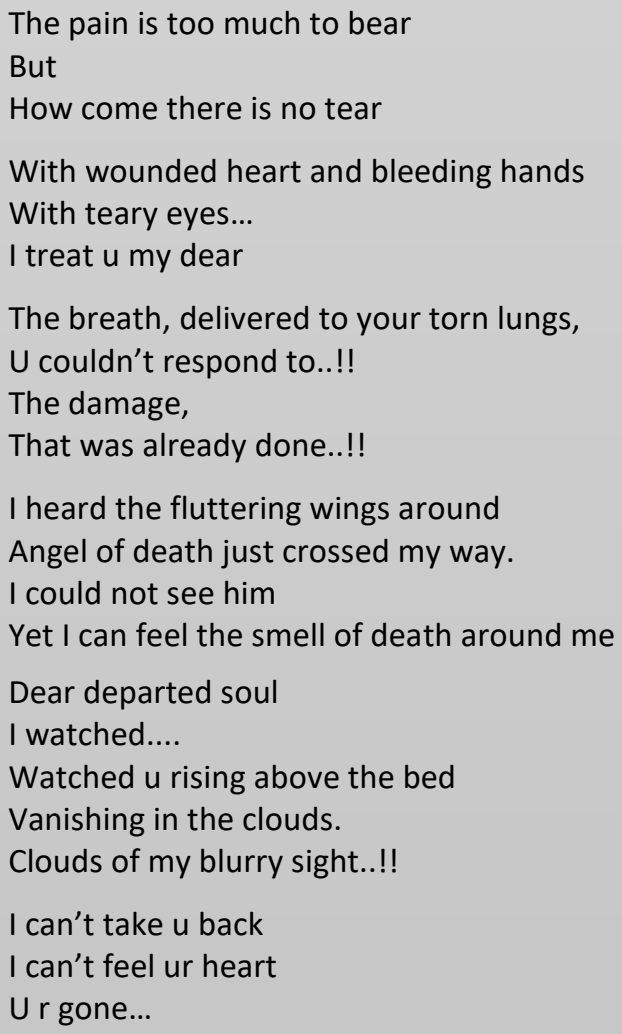

